\title{
The Effect of Breeding Habitat Characteristics on the Larval Abundance of Aedes Vector Mosquitoes (Diptera: Culicidae) in Three Localities, Galle District, Sri Lanka
}

\author{
D. S. Dissanayake, ${ }^{1}$ C. D. Wijekoon $\mathbb{D}^{2},{ }^{2}$ and H. C. Wegiriya ${ }^{2}$ \\ ${ }^{1}$ The Office of the Regional Director of Health Services, Galle, Sri Lanka \\ ${ }^{2}$ Department of Zoology, Faculty of Science, University of Ruhuna, Matara, Sri Lanka \\ Correspondence should be addressed to C. D. Wijekoon; chandanadammika1984@gmail.com
}

Received 16 March 2021; Revised 26 May 2021; Accepted 26 June 2021; Published 8 July 2021

Academic Editor: Luciano Toma

Copyright (c) 2021 D. S. Dissanayake et al. This is an open access article distributed under the Creative Commons Attribution License, which permits unrestricted use, distribution, and reproduction in any medium, provided the original work is properly cited.

\begin{abstract}
Dengue has become a national burden in Sri Lanka, and the understanding of breeding ecology of vectors, Aedes aegypti Linnaeus and Aedes albopictus Skuse, is the most effective way to control the disease. The present study was undertaken to investigate the relative larval abundance of Ae. aegypti and Ae. albopictus in different types and characteristics of containers in three selected localities in Galle district, Sri Lanka. Totally, 550 containers were positive for both Ae. aegypti and Ae. albopictus larvae. Aedes albopictus showed the high larval abundance in all studied sites. The larval abundance of artificial containers (90.57\%) was high than that of natural containers $(9.43 \%)$ for both Aedes spp. $(P<0.05)$. The breeding preference for A. aegypti was high in tires (rubber) (17.82\%), while plastic cups (28.00\%) were the most preferable container type for Ae. albopictus. Dark color containers than light color containers $(P<0.05)$ and containers with leaf litter accumulated as detritus $(P<0.05)$ showed high relative larval abundance for both Aedes species. Containers with 50-100 ml volume of water showed the highest relative abundance of both Ae. aegypti (29.28\%) and Ae. albopictus (41.79\%) $(P>0.05)$. The high larval abundance of Aedes recorded in ground level containers $(1-5 \mathrm{~cm})$ and their abundance decreased significantly with the increasing of height where containers were found $(1-20 \mathrm{~cm})$ $(P<0.05)$. The significantly high relative abundance was observed with the increase of the shady level for Ae. aegypti $(57.34 \%)$ and Ae. albopictus $(61.32 \%)(P<0.05)$. This knowledge will be helpfull to implement dengue surveillance programs in the area.
\end{abstract}

\section{Introduction}

Dengue is one of the most prominent mosquito-borne diseases with a greatest public health importance in the Southeast Asian region. Sri Lanka also has a long history of mosquito vector-borne diseases from time to time, and recently, dengue is considered as the major public health hazard in the country. Since the first dengue outbreak was reported from Sri Lanka in 1965, a dramatic increase of the number of dengue cases has been created being a nationwide burden in the country. Galle district is one of high-risk areas in Southern Province of Sri Lanka, which is continuously affected by dengue in past several years $[1,2]$.

Aedes aegypti Linnaeus and Aedes albopictus Skuse are major vectors of dengue fever (DF) and dengue hemorrhagic fever (DHF). Both species are container-inhabiting mosquitoes [3-6], and Ae. aegypti is the main vector of DF, while Ae. albopictus is the major secondary vector [7]. Both Ae. aegypti and Ae. albopictus are highly anthropophilic daytime biters, and they are dominant in domestic and peridomestic environments [4]. Aedes aegypti is found mainly in urban areas [8], and Ae. albopictus is found mainly in semiurban and rural areas $[5,7,9]$. However, the larvae of both species are found together in the same habitat [10].

The problem with rapid accumulation of discarded containers everywhere increases the difficulties to effective and efficacious Aedes vector control activities. Knowing the most productive container habitats and preferable container characteristics for breeding of Aedes vectors is most important for the implementation of successful vector control 
strategies. As the same, it will support to reduce unnecessary usage of larvicides and adulticides.

The previous studies have been reported the breeding habitats and seasonal preferences of Aedes vector mosquitoes in other countries [3-6] and in Sri Lanka [7]. However, a little is known on the preferable container characteristics for breeding of Aedes vectors in Sri Lanka.

Hence, the current study was started with the intention to investigate the most preferable container characteristics for the breeding of Aedes vector mosquitoes by carrying out larval surveys in three selected sites in Galle district, Sri Lanka.

\section{Materials and Methods}

2.1. Sites Selection. The study was carried out in randomly selected three sites categorized as urban, semiurban, and rural in Galle District, Sri Lanka. Karapitiya GN (Grama Niladhari) area (N 6 $4^{\prime} 14.0952^{\prime \prime}$, E $80^{\circ} 13^{\prime} 30.576^{\prime \prime}$ ), one of the urbanized areas in Galle district, was selected as the urban site, Madampagama GN area ( $\mathrm{N}^{\circ} 2^{\prime} 16.8^{\prime \prime}$, E 80 $06^{\prime}$ $71^{\prime \prime}$ ) was selected as the semiurban site, and Lankagama GN area $\left(\mathrm{N} 6^{\circ} 20^{\prime} 59.99^{\prime \prime}\right.$, E $\left.80^{\circ} 27^{\prime} 59.99^{\prime \prime}\right)$ considered as the rural site (Figure 1).

2.2. Sampling Design. Each site was divided into blocks as fifty homes for each block. A single block was selected at random for each site. Each residence within the selected block was sampled, skipping and recording those residences where permission was not granted. All the potential containers in both indoor and two meters area of the outdoor were examined. Nine larval surveys were conducted as three surveys per each site from January to October in 2017.

2.3. Sampling Procedure. Containers were defined as anything natural or artificial that in its present state was holding water and was capable of holding water for three or more days. All artificial and natural containers were sampled. Pipettes were used to collect all water from containers, and all larvae were placed in individual $50 \mathrm{ml}$ cups with caps, then they labeled (date, place, indoor or outdoor, and sample number) and transported to the laboratory. The sampling variables were recorded for each container at the site using the type of the container and type of the material such as plastic, woody, plant, clay, cement, ceramic, glass, metal, rubber, and rocky. Inner surface color of the container (black, brown, green, yellow, red, gray, and colorless), water depth in the container, water volume, condition of the location (grass, bare soil, pavement, groundcover, and bush), height to base of the container from ground, sun exposure (full sun, partial sun, and full shade), types of detritus within the water (leaves, dirt, or nothing), and the presence and abundance of each Aedes species with each container characteristics were recorded. All collected larvae were counted and identified using available identification keys up to the species level [11]. The mix breeding containers found with both Aedes species during the study were very low, and hence, those data were excluded.
2.4. Data Analysis. Relative abundance of both Aedes species was calculated for different container characteristics. Data were analyzed using IBM SPSS statistics data editor 20.0 version. The one way ANOVA test was applied to test whether there is a significant difference in larval abundance and container characteristics. The level of significant was at $a=$ 0.05. In addition, the chi-square test was applied to check the association between larval abundance and the habitat types.

\section{Results}

Totally, 1067 containers (346 containers in urban, 367 containers in semiurban, and 356 containers in rural areas) were examined. Totally, 550 containers were positive for Ae. aegypti and Ae. albopictus. Among them, 245 containers were positive for the urban site, 210 containers were positive for the semiurban site, and 95 containers were positive for the rural site. In this study, we concerned only these two Aedes species (Ae. aegypti and Ae. albopictus) during the larval survey.

3.1. Comparative Larval Abundance of Aedes spp. among Three Selected Localities. 212 larval mosquitoes of Ae. aegypti and 1434 of Ae. albopictus were recorded from total 5463 in the urban site. 109 specimens of Ae. aegypti and 1606 specimens of Ae. albopictus were recorded from total 3457 specimens of all mosquitoes in the semiurban site. 406 specimens of Ae. albopictus were recorded from 1756 specimens in the rural site. The larval abundance of Ae. aegypti was $3.88 \%$ in urban and $3.15 \%$ in semiurban. There were no records of Ae. aegypti from the rural site. The larval abundance of Ae. albopictus was $46.46 \%, 26.25 \%$, and $23.12 \%$ in semiurban, urban, and rural sites, respectively (Figure 2).

Aedes aegypti showed comparatively similar larval abundance in both urban and semiurban localities, while indicating significantly low larval abundance in the rural site (ANOVA, $P=0.000$ ). There is a significant association between larval abundance of Ae. aegypti and locality types (chi-square test, $P=0.000$ ).

Figure 2 shows the highest larval abundance of $A e$. albopictus from the semiurban site. They were commonly distributed in both urban and rural sites, with no significance (ANOVA, $P=0.899$ ). The association of Ae. albopictus larval abundance with different localities is significant (chi-square test, $P=0.000$ ).

3.2. Occurrence of Aedes spp. in Different Container Types. The larval density was significantly high in artificial containers (90.57\%) than that of natural containers (9.43\%) for both Ae. aegypti and Ae. albopictus (chi-square test, $P=$ 0.000 and 0.005 , respectively).

Table 1 indicates the occurrence of Aedes species in different types of natural and artificial containers recorded from three selected localities.

Among the selected types of containers, the high larval abundance of Ae. aegypti was recorded in tires (rubber) (17.82\%), plastic cups (17.09\%), and the metal cups (9.82\%), but not statistically significant between each container types 

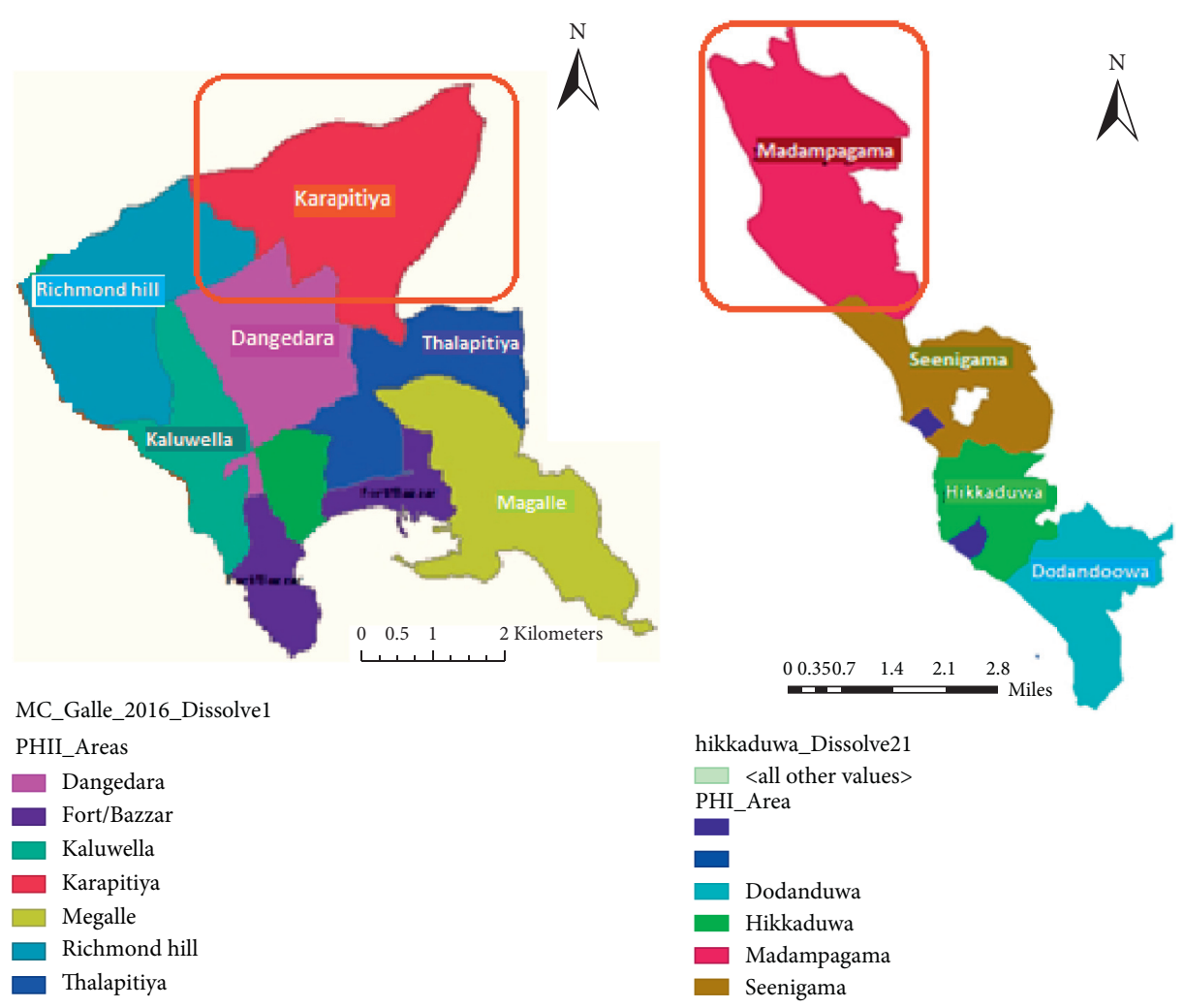

hikkaduwa_Dissolve21

$\square$ <all other values> PHI_Area

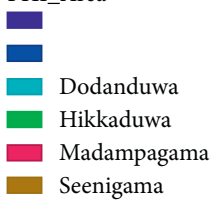

(a)

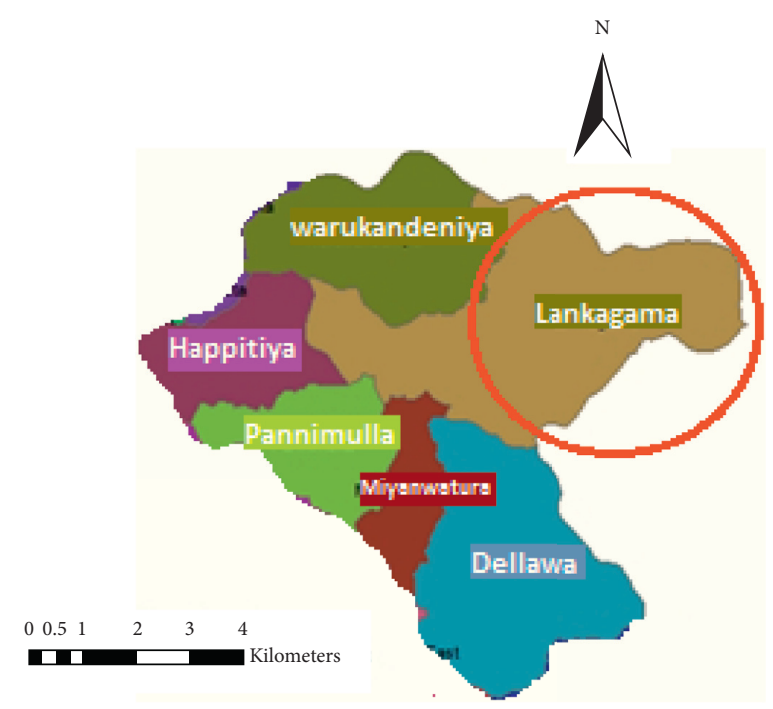

(c)

Figure 1: The selected sites in the Galle district, Sri Lanka. (a) Urban-Karapitiya. (b) Semiurban-Madampagama. (c) Rural-Lankagama.

(ANOVA, $P=0.123$ ). Among the natural container types, plant axils were the most important for larval abundance of Ae. aegypti. The larval abundance in both species was low in rock pools and wood caves (Figure 3).
Plastic cups recorded 28\% relative larval abundance for Ae. albopictus as the highly preferable container type, but not statistically significant (ANOVA, $P=0.222$ ). The comparative larval abundance of Ae. albopictus was less in tires than 


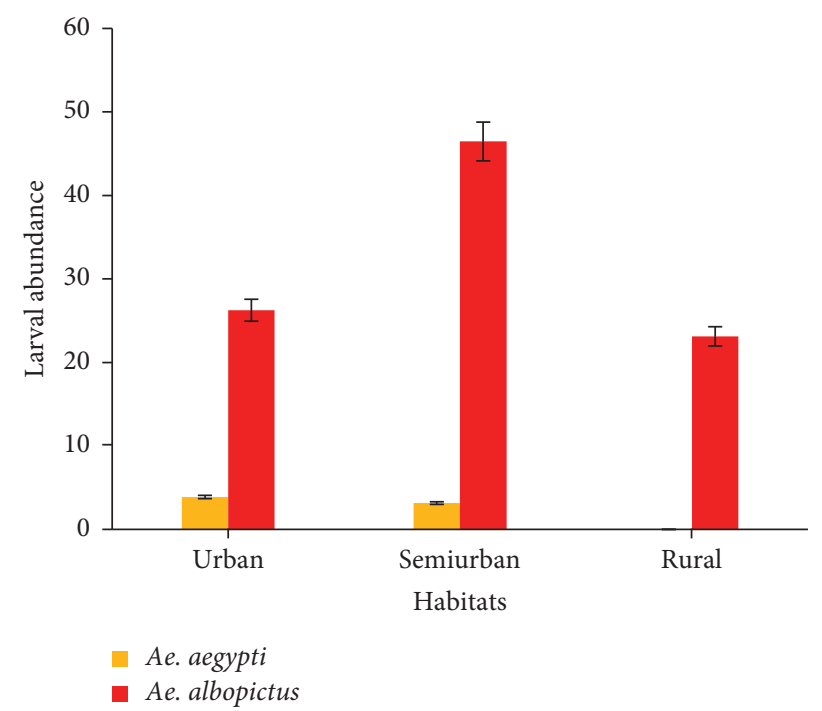

FIgURE 2: Relative abundance of Aedes spp. larvae in three selected habitats.

Table 1: Occurrence of Aedes aegypti and Aedes albopictus in different container types.

\begin{tabular}{|c|c|c|c|}
\hline Type of containers & Type of material & Aedes aegypti & Aedes albopictus \\
\hline \multirow{7}{*}{ Natural } & Animal foot print & - & - \\
\hline & Burrow pits & - & - \\
\hline & Clay pots & + & + \\
\hline & Coconut shells & + & + \\
\hline & Wood caves & + & + \\
\hline & Rain water pools & - & - \\
\hline & Rock pools & + & - \\
\hline \multirow{12}{*}{ Artificial } & Covering items & + & + \\
\hline & Earth pipes & - & - \\
\hline & Flower pots & + & + \\
\hline & Glass boxes & + & + \\
\hline & Metal cups & + & + \\
\hline & Ornamental items & + & + \\
\hline & Pet feeding cups & + & + \\
\hline & Plant axils & + & + \\
\hline & Plastic cups & + & + \\
\hline & Quarry pits & - & - \\
\hline & Toys & + & + \\
\hline & Tires & + & + \\
\hline
\end{tabular}

that of Ae. aegypti. Aedes albopictus also indicated the less larval abundance in wood caves and rock pools (Figure 4).

\subsection{Relative Abundance of Aedes Larvae in Containers with Different Colors, in Different Heights, in Different Volumes of Water, and with Different Types of Detritus.}

3.3.1. Relative Abundance of AedesLarvae in Containers with Different Colors. Black color containers showed the highest relative abundance for Ae aegypti (47.98\%) compared with containers of other colors, but not statistically significant (ANOVA, $P>0.05$ ). Aedes albopictus (37.32\%) also indicated the highest relative larval abundance in black color containers, but not significant (ANOVA, $P>0.05$ ). Brown color containers recorded the second high larval abundance for both Ae. aegypti and Ae. albopictus. The larval abundance of both Aedes species was significantly high in dark color containers than that of light color containers (yellow and colorless) ( $t$-test, $P<0.05$ ) (Figure 5).

3.3.2. Relative Abundance of AedesLarvae in Containers in Different Heights. Aedes aegypti showed high larval abundance in containers that are located at less than $10 \mathrm{~cm}$ heights from the ground. Aedes albopictus indicates a high relative larval abundance in containers found in between 1 and $5 \mathrm{~cm}$ heights. Interestingly, figure 6 shows that the both Aedes spp. record their high larval abundance in ground level containers $(1-5 \mathrm{~cm})$ and the larval abundance significantly decrease when increasing the height of the containers found $(1-20 \mathrm{~cm}) \quad($ ANOVA, $\quad P<0.05)$ (Figure 6). 


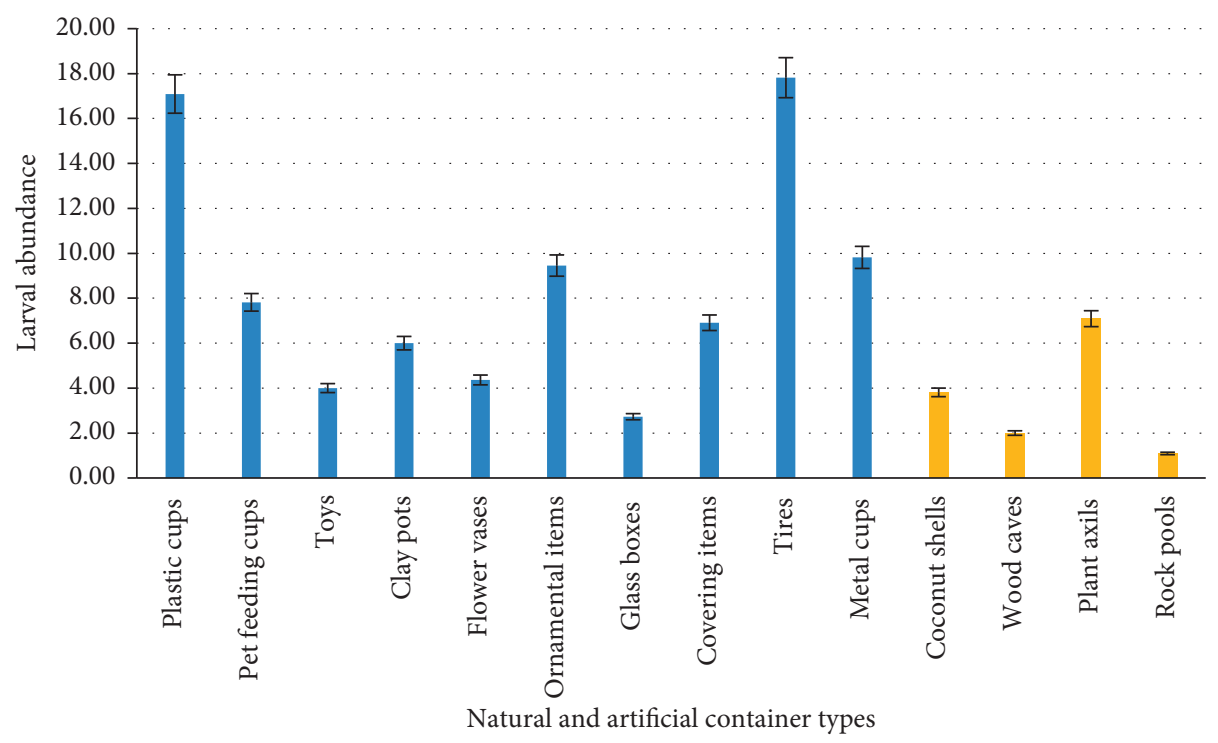

FIgURE 3: Relative abundance of Aedes aegypti larvae in natural containers and artificial containers.

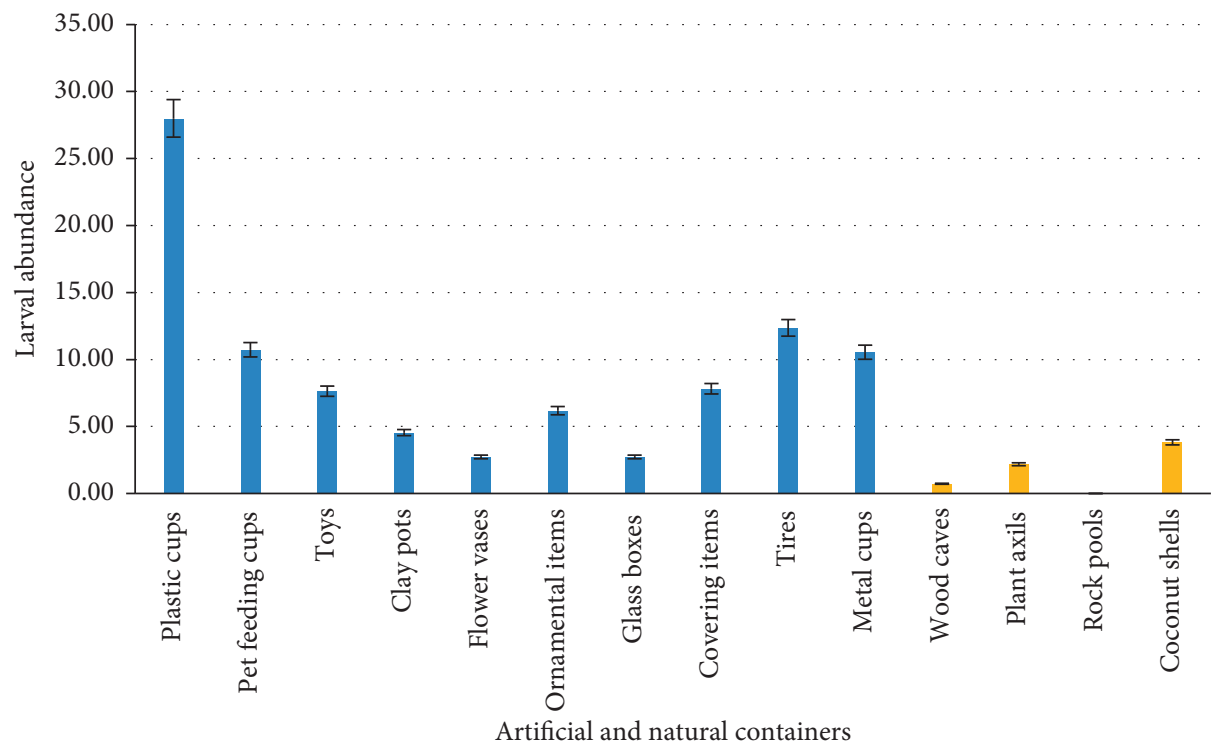

Figure 4: Relative abundance of Aedes albopictus larvae in natural containers and artificial containers.

3.3.3. Relative Abundance of AedesLarvae in Containers in Different Volumes of Water. Containers with $50-100 \mathrm{ml}$ volume of water indicated the highest relative larval abundance of both species (29.28\% Ae. aegypti and $41.79 \%$ Ae. albopictus) (ANOVA, $P>0.05$ ). Both species showed very low larval abundance when the water level becomes very low in containers or when increases more than $150 \mathrm{ml}$ (Figure 7).

3.3.4. Relative Abundance of Aedes Larvae in Containers in Different Shady Levels and Different Types of Detritus. The relative abundance of Ae. aegypti and Ae. albopictus was high in containers located in fully shady places (Figure 8 ). Their relative abundance increases with high shady level (57.34\% Ae. aegypti and $61.32 \%$ Ae. albopictus). There is a statistical significance of larval abundance of Ae. aegypti between containers found in full shade locations and full sun locations (ANOVA, $P=0.049$ ). Aedes albopictus larval abundance is significantly different in containers found from full shady locations and from full sunny locations (ANOVA, $P=0.000$ ).

The different types of detritus occurred in each containers indicated that the larval abundance of both $A e$. aegypti and Ae. albopictus was significantly high when leaf litter presents as the detritus in the containers $(80.69 \% A e$. aegypti (ANOVA, $P=0.020$ ) and $73.77 \%$ Ae. albopictus (ANOVA, $P=0.037)$ ). Their larval abundance is very low when sand occurs as the detritus in the container (Figure 9).

\section{Discussion}

It is well known that Ae. aegypti and Ae. albopictus are main vectors of DF and DHF in Sri Lanka and both are container- 


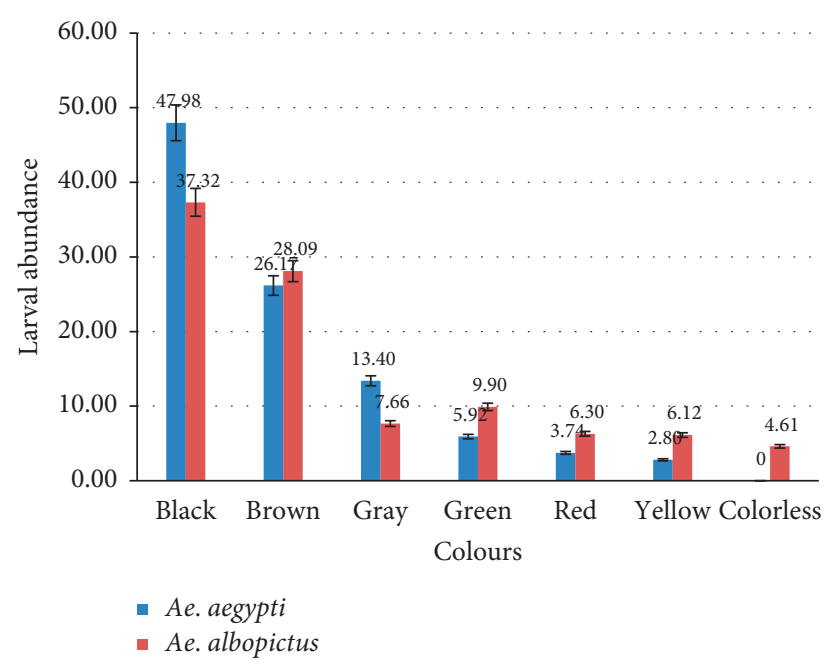

Figure 5: Relative larval abundance of Aedes spp. in containers with different colors.

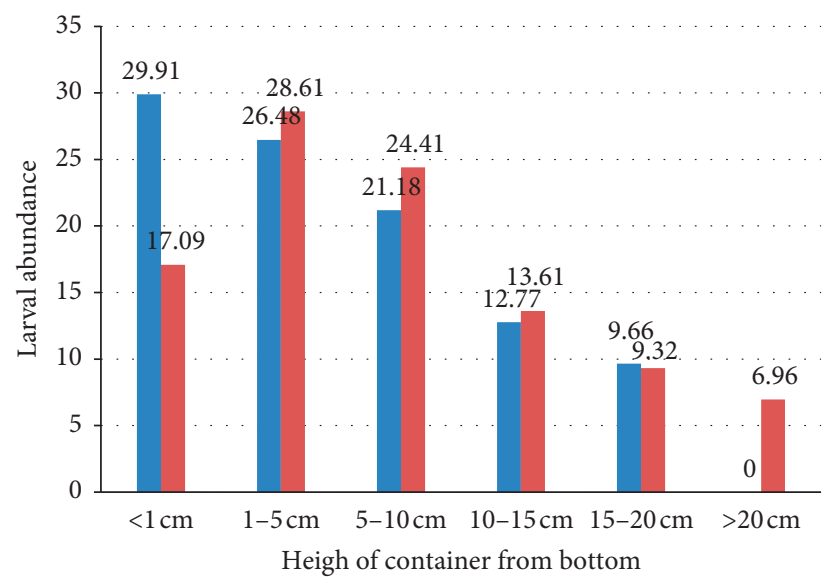

$$
\begin{aligned}
& \text { - Ae. aegypti } \\
& \text { - Ae. albopictus }
\end{aligned}
$$

Figure 6: Relative abundance of Aedes spp. larvae in containers with different heights.

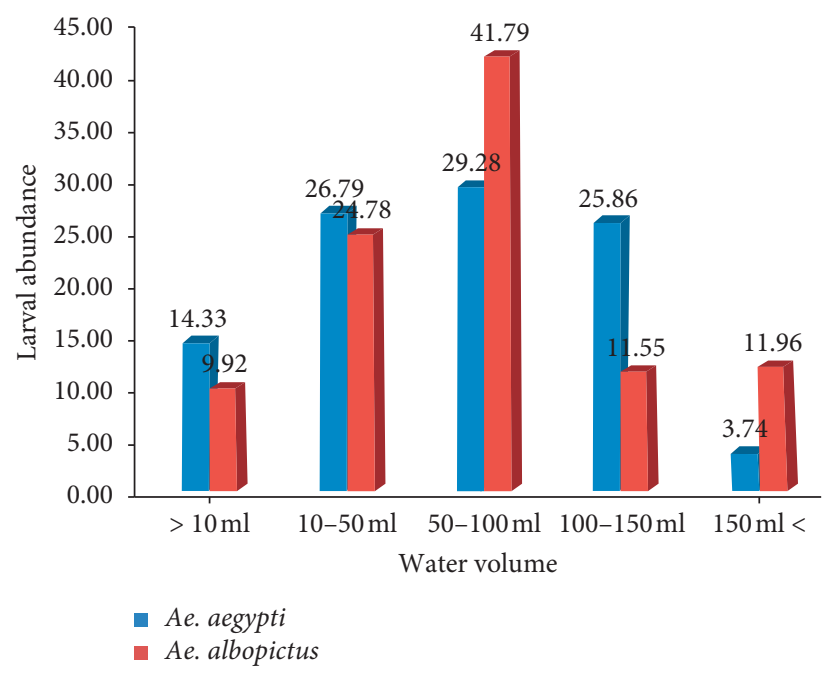

FIgURE 7: Relative abundance of larvae in containers with different volumes of water. 


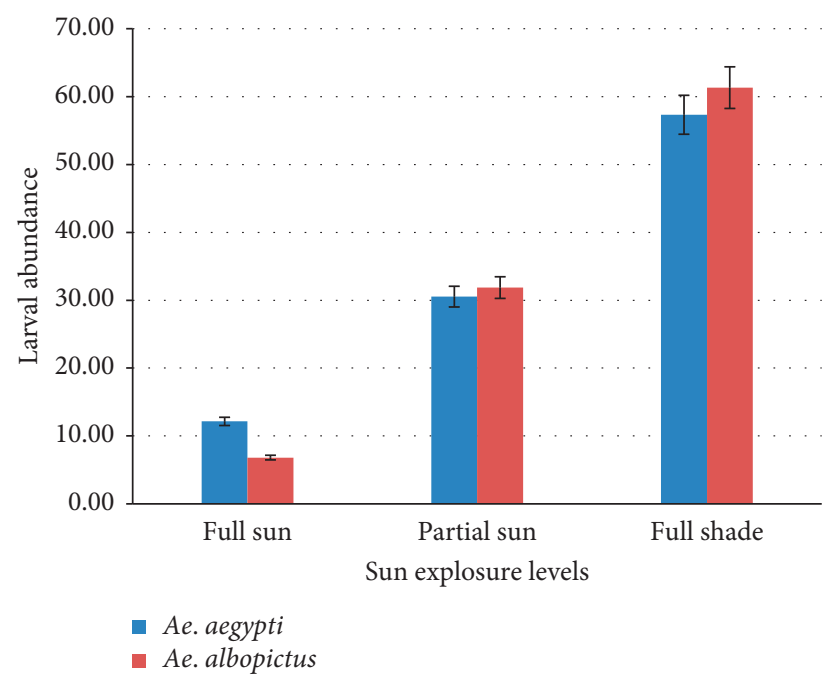

Figure 8: Relative abundance of larvae in containers with different shady levels.

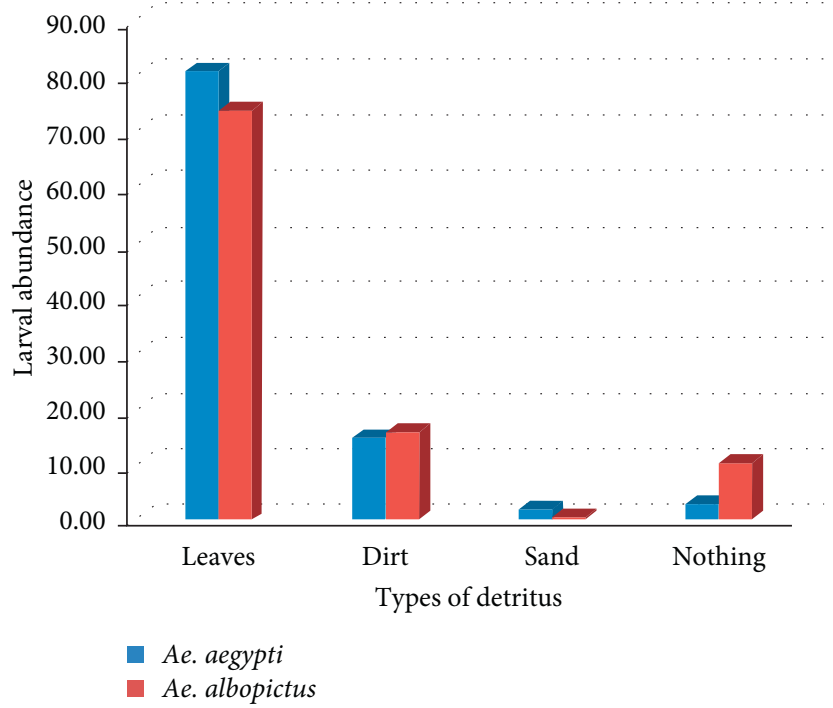

FIGURE 9: Relative abundance of larvae in containers with different types of detritus.

inhabiting mosquitoes that deposit eggs in small type of containers. The present study reveals the effects of container characteristics on the breeding preference of larvae of Aedes vector mosquitoes across the selected urban, semiurban, and rural sites in Galle district, Sri Lanka.

Aedes albopictus is the dominant species in all selected sites of the present study. Aedes aegypti is less abundant in urban and semiurban, and there are no records in the rural site. A notable deduction in the population of Ae. aegypti in rural sites has been reported by several previous studies [7, 12-15]. In 2012, Bartlett-Healy et al. further implied that Ae. aegypti is an urban mosquito species while Ae. albopictus prefers semiurban environments and breeds in outdoor natural breeding sites.

In the present study, we excluded the mix breeding sites which found both Aedes spp. in same habitat because they were recorded in few numbers.
Compared with the number of positive containers encountered, the numbers of artificial containers are high than that of natural containers, indicating the possible risk of the spread of disease by rapid accumulation of discarded artificial containers in the study area. A study conducted in Central Africa has shown that artificial containers were most frequently encountered than natural containers for both Ae. aegypti and Ae. albopictus [4]. Furthermore, in 2017, Julien et al. recorded that the predominant breeding sites were industrial containers for Aedes vectors in the urban setting in southeastern Côte d'Ivoire.

Among the positive artificial containers recorded, tires (rubber) are the most preferable artificial breeding site for Ae. aegypti, while plastic cups are the most preferable artificial breeding site for Ae. albopictus. Tires and plastic cups are commonly found artificial discarded materials in all sites during the study. Used tires and plastic containers are the predominantly identified industrial containers $[4,5,16]$. 
Our study shows that dark color containers have significantly high breeding preference for both Aedes spp. than that of light color containers. Dark and cool resting places provide the need of mosquito life cycle [17], black and gray with the species presence for Ae. albopictus and Ae. japonicus [16]. However, controversy results in the study by [3] presented that Ae. aegypti and Ae. albopictus larvae were commonly found more in light color containers in Thailand.

Significantly, we found that both Ae. aegypti and Ae. albopictus record their high larval abundance in ground level containers $(1-5 \mathrm{~cm})$ and significantly decrease the larval abundance when increasing the height where containers were found $(1-20 \mathrm{~cm})$. As well as, the containers with $50-100 \mathrm{ml}$ water level indicate the highest breeding preference for both Aedes species, and their larval abundance becomes very low when the water level increases more than $150 \mathrm{ml}$. In 2016, Kabirul et al. mentioned that most of Aedes species select shallow water depth for their oviposition.

Containers found in shaded areas provide ideal habitats for mosquito larvae. Natural shade not only lowers water temperatures but often provides a bacterial food source for mosquito larvae from fallen leaves and debris that are subsequently collected in containers [18]. The abundance of Ae. albopictus larva in shaded or partially shaded areas may be attributed in part to abundant food resources in shaded containers. In the present study, the high relative abundance of Ae. aegypti and Ae. albopictus was observed in containers of full shady habitats. Containers that expose to direct sunlight show less larval abundance of both Aedes mosquitoes.

The study reveals a significant high larval abundance of both Aedes species in containers where leaf litter occurs as detritus. Several previous authors addressed that the leaf litter provides an important nutrient source for bacteria, which in turn is the primary food source for mosquito larvae $[19,20]$, and Ae. albopictus prefers to oviposit in containers with leaf litter $[16,21,22]$.

The present study provides the most important information on the container characteristics of the breeding preference of Ae. aegypti and Ae. albopictus, main dengue vectors in Sri Lanka. The study shows that the artificial discarded containers were the most preferred type of breeding habitat in all studied sites, and the accumulation of these containers make serious future health risk for dengue in the study area. Hence, study findings would be helpful for researchers and health authorities to design appropriate vector control measures and to mitigate future dengue outbreaks in the area.

\section{Conclusions}

Aedes albopictus is the dominant dengue vector species found from all study sites (urban, semiurban, and rural) in the Galle district of Sri Lanka. Artificial containers are more productive for the oviposition of Ae. aegypti and Ae. albopictus than natural containers. Tires are the most preferable artificial breeding site for Ae. aegypti. Aedes albopictus selects plastic cups as the most preferable artificial breeding site. Dark color containers are more favorable that contained with water between $50 \mathrm{ml}$ and $100 \mathrm{ml}$ volume and locate less than $5 \mathrm{~cm}$ height from the ground level for the optimum breeding of Ae. aegypti and Ae. albopictus. Presence of leaf litter as detritus in the container and the increase of the shady level, positively support to the highest productivity of Ae. aegypti and Ae. albopictus. The findings of the study help in the effective and efficacious Aedes control and management activities in epidemic situations for public health aspects.

\section{Data Availability}

The datasets used to support the findings of this study are available from the corresponding author upon request.

\section{Conflicts of Interest}

The authors declare that they have no conflicts of interest.

\section{Authors' Contributions}

Dinithi S. Dissanayake conducted field surveys, data collection, and data entering. Chandana D. Wijekoon analyzed the data and wrote the manuscript. Hemantha C. Wegiriya supervised the research and reviewed the manuscript.

\section{Acknowledgments}

The authors would like to thank all field supporters for their kind help to collect various information for the study.

\section{References}

[1] The Ministry of Health, Guidelines for Aedes Vector Surveillance and Control, National Dengue Control Unit, Ministry of Health and nutrition, p. 11, The Ministry of Health, Colombo, Sri Lanka, 2016.

[2] World Health Organization, The Dengue Strategic Plan for the Asia Pacific Region 2008-2015, WHO, vol. 8, p. 53, Geneva, Switzerland, 2008.

[3] A. Chumsri, F. W. Tina, M. Jaroensutasinee, and K. Jaroensutasinee, "Seasons and socio-cultural practices affecting Aedes mosquito larvae in southern Thailand," Tropical Biomedicine, vol. 35, no. 1, pp. 111-125, 2018.

[4] S. Frédéric, N. Elysée, T. Jean Claude, and F. Didier, "Geographic distribution and breeding site preference of Aedes albopictus and Aedes aegypti (Diptera: culicidae) in Cameroon, Central Africa," Journal of Medical Entomology, vol. 42, no. 5, pp. 726-731, 2005.

[5] B. Z. Julien, B. G. Koudou, P. Muller, D. Malone, Y. Tano, and J. Utzinger, "Urbanization is a main driver for the larval ecology of Aedes mosquitoes in arbovirus-endemic settings in south-eastern Côte d'Ivoire," PLoS Neglected Tropical Diseases, vol. 11, no. 7, Article ID e0005751, 2017.

[6] A. R. Khan, "Studies on the breeding habitats and seasonal prevalence of larval population of Aedes aegypti (L.) and Aedes albopictus (Skuse) in Dacca city," Bangladesh Med Res Counc Bull, vol. 6, no. 2, pp. 45-52, 1980.

[7] C. T. Weerarathne, M. D. B. Perera, M. A. C. M. Mansoor, and S. H. P. P. Karunarathne, "Prevelance and breeding habitats of dengue vectors Aedes aegypti and Aedes albopictus (Diptera: Culicidae) in the semi-urban areas of two different climate 
zones in Sri Lanka," International Journal of Tropical Insect Science, vol. 33, no. 4, pp. 216-226, 2013.

[8] S. R. Christophers, Aedes aegypti the Yellow Fever Mosquito, its Life Story Bionomics and Structure, p. 60, Cambridge University Press, London, UK, 1960.

[9] W. A. Hawley, "The biology of Aedes albopictus," Journal of American Mosquito Control Association, vol. 4, pp. 1-40, 1988.

[10] M. A. Braks, N. A. Honorio, R. Lourenco-De-Oliveira, S. A. Juliano, and P. Lounibos, "Convergent habitat segregation of Aedes aegypti and Aedes albopictus (Diptera: culicidae) in southeastern Brazil and Florida," Journal of Medical Entomology, vol. 40, pp. 785-794, 2003.

[11] F. P. Amerasinghe, "Catalogue of the mosquitoes of Sri Lanka. Natural resources, energy and science authority of Sri Lanka," p. 23, 1991.

[12] D. Fontenille and J. C. Toto, "Aedes albopictus, a potential new dengue vector in Southern Cameroon," Emerging Infectious Diseases, vol. 7, pp. 1066-1067, 2001.

[13] J. H. Hobbs, E. A. Hughes, and B. H. Eichold, "Replacement of Aedes aegypti by Aedes albopictus in mobile, Alabama," Journal of American Mosquito Control Association, vol. 7, pp. 488-489, 1999.

[14] G. F. Meara, L. F. Evans, D. G. Alan, and J. P. Cuda, "Spread of Aedes albopictus and decline of Aedes aegypti (Diptera: culicidae) in Florida," Journal of Medical Entomology, vol. 32, pp. 554-562, 1995.

[15] C. G. Moore, "Aedes albopictus in the United States; current status and prospects for further spread," Journal of American Mosquito Control Association, vol. 15, pp. 221-227, 1999.

[16] K. Bartlett-Healy, I. Unlu, P. Obenauer et al., "Larval mosquito habitat utilization and community dynamics of Aedes albopictus and Aedes japonicus (Diptera: Culicidae)," Journal of Medical Entomology, vol. 49, no. 4, pp. 813-824, 2012.

[17] A. G. Sarah, C. M. Amy, L. B. Jose et al., "River boats contributeto the regional spread of the dengue vector Aedes aegypti in the Peruvian Amazon," PLoS Neglected Tropical Diseases, vol. 9, pp. 4-13, 2015.

[18] J. S. Eaton, G. E. Likens, and F. H. Bormann, "Through fall and stem-flow chemistry in northern hardwood forest," Journal of Ecology, vol. 61, pp. 495-508, 1973.

[19] R. W. Merritt, R. H. Dadd, and E. D. Walker, "Feeding behaviour, natural food, and nutritional relationships of larval mosquitoes," Annual Review of Entomology, vol. 37, pp. 349-376, 1992.

[20] Y. Tsuda, M. Takagi, and Y. Wada, "Ecologicalstudyon mosquito community in tree holes in Nagasaki, Japan, with reference to Aedes albopictus (Diptera: Culicidae)," Medical Entomology and Zoology, vol. 45, pp. 103-111, 1994.

[21] H. Dieng, M. Boots, Y. Tsuda, and M. Takagi, "A laboratory oviposition study in Aedesalbopictus (Diptera: Culicidae) with reference to habitat size, leaf litter and their interactions," Medical Entomology and Zoology, vol. 54, pp. 43-50, 2003.

[22] L. P. Lounibos, "Invasions by insect vectors of human disease," Annual Review of Entomology, vol. 47, pp. 233-266, 2002.

[23] B. Kabirul, M. D. S. Rahman, J. Nodi, and A. J. Howlader, "Species composition and habitat characterization of mosquito (Diptera: Culicidae) larvae in semi-urban areas of Dhaka, Bangladesh," Journal of Pathogens of Global Health, vol. 110, no. 2, pp. 48-61, 2016. 